\title{
THE COSMOS PLATE MEASURING MACHINE
}

\author{
N. M. PRATT \\ Royal Observatory, Edinburgh, U.K.
}

\section{Introduction}

The new 48-in. Schmidt telescope of the U.K. Science Research Council is being commissioned at Siding Spring in Australia. Its potential both for extra-galactic and galactic research is enormous but this potential can only be fully exploited with an automatic measuring machine.

Using the prototype GALAXY machine as a basis, discussions were begun with FCS Controls Ltd, of Haddington, Scotland, in 1969 and have resulted in a new machine which is scheduled to have its acceptance trials in September 1973.

\section{The New Machine}

The only parts of GALAXY which have been retained in the new machine are the plate carriage, with its associated hydraulic and Moiré fringe position systems, and the two basic scanning systems. The special purpose electronics, including the servo systems, have been redesigned and a Honeywell 316 computer has been introduced to supervise the operation of the machine.

The raster scanning system provides the means for a rapid survey of plates for galaxies, stars and nebulae with approximate measures; it can also be used in a transmission mapping mode for high resolution studies of galaxies and nebulae. The spiral scanning system has been modified so that it can make precise measurements of position and size of both galaxies and stars. This has been achieved by generalising the scanning pattern from circles to ellipses whose eccentricity is automatically matched to that of the object being measured. This system can also be adapted to the measurement of objective prism spectra.

The plate carriage can accommodate plates up to $350 \mathrm{~mm}$ sq and all output is to magnetic tape.

\section{Coarse Measurement Mode}

The spot size of the raster scan on the plate can be either 8,16 or $32 \mu \mathrm{m}$, the width of the raster lane being 128 times the spot diameter. The output for each image detected is:

$X$ and $Y$ coordinates;

the area of the image;

the $X$ extent;

the $Y$ extent; 
the minimum transmission in terms of a greyness scale with approximately 90 levels;

the quadrant of orientation.

This information may be sufficient to separate stars from galaxies and other objects. The positions of 'good' images are accurate to a fraction of the spot size.

The estimated rate of scanning with the $8 \mu \mathrm{m}$ spot is $2 \mathrm{~mm}^{2} \mathrm{~s}^{-1}$, or one plate $350 \times 350 \mathrm{~mm}$ in 17 hours. With the $16 \mu \mathrm{m}$ spot the rate of scan is four times faster, but with lower resolution. The data for approximately $10^{6}$ images can be stored on one magnetic tape.

\section{Mapping Mode}

The raster scan can also be used in a digital transmission mapping mode. In this mode the transmission through each spot size element is integrated and digitised in terms of the greyness scale. Scanning is at the same rate as in coarse measurement mode. With the $8 \mu \mathrm{m}$ spot, the data from approximately $1500 \mathrm{~mm}^{2}$ of plate can be stored on one magnetic tape.

\section{Fine Measurement Mode}

The direct computer control permits greater versatility in the profile matching system. In addition to the normal all image technique, it is possible to measure profiles in narrow transmission ranges thus giving a contour mapping facility in the form of best fitting ellipses at specified transmission levels. It is also possible to measure the transmission in an annulus outside an image.

The bit size of the $X$ and $Y$ coordinates has been reduced from $1 \mu \mathrm{m}$ to $0.5 \mu \mathrm{m}$. Three interchangeable optical systems are available enabling different ranges of image size to be measured.

\section{Spectrum Scanning Mode}

For measuring spectra, the spiral scan is given the form of an elongated ellipse. The extremities of the major axis are used to centre on the spectrum while the minor axis is used to measure either the transmission at intervals along the spectrum, or the positions and strengths of spectral lines.

\section{DISCUSSION}

Klock: Is the figure you quoted of $\pm 0.5 \mu$ for position to be interpreted as repeatability? Murray: It is the least count of read out.

Klock: What is the resolution limit of the machine, that is, how close may two images be and still be successfully measured?

Murray: Stars with a clear separation of at least one scan increment will certainly be resolved. 